\title{
Actinobacillus Actinomycetemcomitans y Porphyromonas Gingivalis como principales patógenos periodontales
}

\author{
BASCONES A * \\ CABALLERO $\boldsymbol{A} * *$
}

Bascones A, Caballero A. Actinobacillus

Actinomycetemcomitans y Porphyromonas Gingivalis como principales patógenos periodontales. $2000 ; 12,2: 69-75$

\begin{abstract}
RESUMEN
Entre las bacterias relacionadas con la enfermedad periodontal, existen dos especies más claramente asociadas a esta enfermedad: Actinobacillus actinomycetemcomitans y Porphyromonas gingivalis. Este trabajo es una revisión bibliográfica sobre estos dos patógenos periodontales, mostrando su origen, prevalencia, distribución, transmisión y respuesta al tratamiento periodontal.
\end{abstract}

\section{PALABRAS CLAVE}

Actinobacillus actinomycetemcomitans, Porphyromonas gingivalis, patógeno periodontal, destrucción periodontal.

La periodontitis como enfermedad periodontal, se caracteriza por la pérdida del aparato de inserción periodontal de los dientes. Este proceso incluye varias entidades que se manifiestan mediante diferentes presentaciones clínicas y mecanismos patogénicos. La periodontitis se considera una enfermedad infecciosa de origen bacteriano, habiéndose descrito en la literatura más de 300 patógenos posiblemente relacionados con la destrucción periodontal.

Se considera patógeno a aquella bacteria capaz de desafiar los mecanismos de defensa del huésped, causar daño y alterar el equilibrio entre el huésped y la microflora oral (1). Según Socransky (2), los criterios para definir los patógenos periodontales incluyen: 1) que exista relación con la enfermedad periodontal con incrementos en la cantidad de microorganismos en sitios enfermos; 2) presencia de mejoría clínica tras la eliminación o disminución de las bacterias del área subgingival; 3) presencia de reacción inmunitaria celular o humoral del huésped; 4) patogenicidad en modelos animales experimentales; y 5) presencia de factores de virulencia que permitan que el microorganismo generen destrucción de los tejidos periodontales

\section{RELACIÓN CON LA ENFERMEDAD PERIODONTAL}

Las infecciones periodontales se consideran infecciones bacterianas mixtas (2), causadas principalmente por bacterias anaerobias gramnegativas. De todos los patógenos periodontales descritos, dos de ellos parece que están más claramente asociados a la periodontitis: Actinobacillus actinomycetemcomitans (Aa) y Porphyromonas gingivalis $(\mathrm{Pg})$.

Aa es un coco o bacilo corto, pequeño, gramnegativo, capnofílico, no-móvil y sacarolítico. En niños sanos, Aa puede llegar a estar presente en un 0-26\% (3). La enfermedad periodontal destructiva está frecuentemente asociada a este patógeno y se considera el causante principal de la periodontitis de inicio precoz. En la periodontitis prepuberal, la prevalencia del $\mathrm{Aa}$ es del 40-100\%. La periodontitis juvenil localizada es la entidad que está más asociada a esta bacteria, la cual se encuentra en un $75-100 \%$ de las lesiones (4). En la periodontitis del adulto está presente sólo en un $30-40 \%$, ya que con la edad disminuye su prevalencia.

\footnotetext{
* Catedrático de Medicina Bucal y Periodoncia. Universidad Complutense de Madrid

** Odontóloga. Práctica privada. Valencia.
} 
$P g$ es un bacilo gramnegativo, anaerobio, no-móvil y asacarolítico, que produce colonias con pigmentaciones marrones en medio de cultivo agar-sangre. Los niños y adolescentes sanos no suelen presentarlo en su microbiota subgingival (2). Se ha descrito en un 37 $63 \%$ de los pacientes con periodontitis juvenil localizada, aunque representa una pequeña parte de la microbiota en los estadios iniciales de la enfermedad. Sin embargo, $\mathrm{Pg}$ es el patógeno principal de la periodontitis juvenil generalizada. En la periodontitis del adulto su prevalencia es del 40-100\%, es el patógeno más importante y se encuentra en mayor proporción en las bolsas profundas $(5,6,7,8)$.

\section{DIAGNÓSTICO MICROBIOLÓGICO}

La periodontitis tiene una etiología microbiana, por lo que, para el diagnóstico etiológico de la enfermedad, es importante la identificación de la microbiota periodontal. Los métodos de detección de la flora oral son complejos y específicos $(9,10)$ y se pueden dividir en función de su metodología, en tres grandes grupos: cultivos selectivos (11), inmunodiagnóstico y técnicas moleculares, que incluyen la técnica de sonda DNA y la reacción en cadena de la polimerasa.

Si se utilizan de forma apropiada, los tests microbiológicos tienen distintas aplicaciones clínicas (12):

- identificar posibles factores de riesgo

- evaluar la severidad de la enfermedad

- determinar el pronóstico

- establecer un plan de tratamiento adecuado

- elegir un antibiótico específico

\section{FUENTE DE PATÓGENOS PERIODONTALES}

Las infecciones bacterianas pueden tener un orígen endógeno o exógeno, es decir, una microflora residente $\circ$ saprófita, o patógenos provinientes del ambiente externo (13). El conocimiento de la fuente de patógenos periodontales y su transmisión entre distintos individuos tiene implicaciones en la prevención y en el tratamiento de la enfermedad.

Un patógeno periodontal exógeno puede ser eliminado completamente de la cavidad oral y se puede transmitir entre individuos; sin embargo, un patógeno periodontal endógeno no puede ser totalmente erradicado de la boca, y ha de ser suprimido mediante tratamientos de repetición mecánicos o químicos. La infección endógena se produce por un sobrecrecimiento bacteriano o por una disminución en las defensas.
Se piensa que los principales patógenos periodontales, $A a$ y $P g$ tienen un orígen exógeno $(13,14,15,16$, $17,18,19)$ debido a que rara vez se encuentran en individuos periodontalmente sanos. Además, se pueden transmitir entre individuos, provocan una respuesta intensa del huésped a la infección y existe la posibilidad de eliminarlos del área subgingival (13). No obstante, hay autores que defienden que las infecciones periodontales son causadas por la microflora oral saprófita y, por tanto, tienen un orígen endógeno $(20,21)$.

\section{DISTRIBUCIÓN EN LA CAVIDAD ORAL}

La distribución de Aa en la cavidad oral es distinta a la de $P g$ y esto se debe a diferentes factores: los requerimientos para el crecimiento, el ambiente ecológico, el modo de transmisión y los factores que alteran el equilibrio huésped-bacteria (22).

El Aa es uno de los primeros colonizadores de la placa bacteriana de las superficies dentales (23), lo que sugiere que puede colonizar superficies limpias y sanas. $\mathrm{Pg}$, por el contrario, no coloniza superficies limpias (24), sino que suele encontrarse en zonas con inflamación, pobre higiene oral y en sitios con placa bacteriana gram-positiva $(24,25,26,27,28,29,30)$.

En relación con la edad, $A$ a suele aparecer en pacientes jóvenes y su presencia disminuye con la edad, mientras que $P g$ aparece más tardíamente, ya que para su crecimiento requiere un entorno que no suele encontrarse en los niños.

El hábitat natural de $A a$ y $P g$ es el área subgingival, ya que requieren para su desarrollo un ambiente anaeróbio como el que proporcionan las bolsas periodontales $(31,32,33,34)$. En el área supragingival también se encuentra en una proporción alta, aunque es en el área subgingival donde encuentran el ambiente idóneo para su crecimiento y desarrollo. Además, estos dos patógenos han sido recogidos de la saliva (31, 33, $35,36,37)$, dorso de la lengua $(31,33,35,38)$, faringe (36) y mucosa oral $(32.34,36,39,40)$. De hecho, la proporción de $A a$ y $P g$ en tejidos blandos es tan alta como en la placa supragingival y en el caso de $\mathrm{Aa}$, tanto como en la subgingival (41).

Los estudios sobre la relación de estos patógenos con la periodontitis indican que hay una mayor asociación de $\mathrm{Pg}$ con los signos de enfermedad periodontal. $\mathrm{Pg}$ se relaciona con inflamación periodontal, aumento de la profundidad de sondaje, pobre higiene oral y pérdida de inserción $(25,26,28,42,43,44,45,46,47,48)$. En cuanto al $A a$, los resultados son contradictorios. Wolff et al. (49) no vieron una diferencia significativa 
en el índice gingival, profundidad de sondaje o nivel de inserción entre cultivos positivos y negativos de Aa. Sin embargo, Ebersole et al. (50) asocian la presencia del patógeno con aumento en la profundidad de bolsas y pérdida de inserción.

\section{EQUILIBRIO HUÉSPED-FLORA}

En el individuo sano, la flora de la cavidad oral está en equilibrio ecológico con el huésped y esto permite mantener en estado de salud las estructuras del periodonto. Pero esta relación estable se puede alterar como consecuencia de una serie de factores como la terapia antimicrobiana o los cambios en la susceptibilidad del huésped debidos a la alteración de algún mecanismo de defensa (1).

En un periodonto sano, $\mathrm{Aa}$ y $\mathrm{Pg}$ pueden estar presentes en proporciones bajas y con un crecimiento controlado. Si aumenta la cantidad de estos patógenos se altera la homeostasis del ecosistema, lo que conduce a la aparición de la enfermedad periodontal. Estudios que comparan la cantidad de $A a$ y $P g$ en la flora subgingival, muestran que predomina la presencia de $\mathrm{Pg}$. Torkko \& Asikainen (51) y Rams et al. (52) encontraron una proporción de $A a$ del $4 \%$, y de $P g$ del $16 \%$ y $23 \%$ respectivamente. Esto significa que la multiplicación de éste último en el área subgingival es más efectiva que la de $A a$ y que los mecanismos de defensa del huésped no son capaces de controlar el crecimiento de $P g$ (53).

\section{TRANSMISIÓN DE PATÓGENOS}

Se han realizado estudios sobre transmisión de patógenos periodontales entre individuos de una misma familia. La transmisión se ha estudiado entre cónyuges, o transmisión horizontal, y entre padres e hijos, o transmisión vertical.

$P g$ es difícil de aislar en niños, por lo que la mayoría de estudios sobre transmisión vertical de patógenos periodontales se han realizado sobre $A a(54,55,56)$. Los resultados muestran que cuando un niño presenta $A a$, los padres suelen tener el mismo genotipo bacteriano de $A a$, lo que sugiere que la transmisión vertical es posible.

Los resultados sobre la transmisión de patógenos periodontales entre cónyuges se contradicen. En estudios sobre la transmisión horizontal, los resultados varian entre un $14 \%$ y un $60 \%$ de transmisión de Aa $(56,57)$ y un $30 \%$ y un $75 \%$ de $P g(56,58)$. Por el contrario, hay estudios que demuestran que la trans- misión de estos patógenos periodontales entre cónyuges es poco frecuente (56). La explicación a estos resultados contrapuestos puede ser que, aunque se puede producir habitualmente transmisión de patógenos periodontales entre cónyuges, no siempre se desarrolla la colonización, ya que ésta depende de una serie de variantes como:

- el huésped

- las características de la cepa

- el número de bacterias inoculadas

- el tiempo de exposición a la infección

El vehículo de transmisión de los patógenos periodontales entre individuos puede ser diverso: la saliva, las mucosas o los objetos inanimados como el cepillo de dientes. El papel de la saliva en la transmisión de Aa ha sido el más estudiado $(31,37,38)$. Parece ser que, dado que el nicho ecológico de $\mathrm{Aa}$ y $\mathrm{Pg}$ es el área subgingival, las bacterias presentes en la saliva deben provenir de las bolsas periodontales, por lo que a mayor cantidad en saliva, mayor riesgo de recolonización. Con el tratamiento periodontal se puede eliminar o disminuir de forma importante la presencia de estas dos bacterias en la saliva, al menos durante seis meses (37). Sin embargo, no parece posible la supresión de estas bacterias de las membranas mucosas.

\section{RESPUESTA AL TRATAMIENTO PERIODONTAL}

Cuando el Aa está presente en un paciente no tratado periodontalmente, puede persistir durante mucho tiempo, lo que indica que los mecanismos de defensa del huésped no lo pueden eliminar (59). El tratamiento de raspado y alisado radicular parece ser insuficiente para erradicar este organismo, ya que el microorganismo tiene la capacidad de invadir los tejidos gingivales (60). Aparentemente, la cirugía resectiva obtiene mejores resultados que la cirugía de acceso, porque se elimina el tejido gingival infectado, se reduce la bolsa periodontal y se permite una mejor higiene oral. Pero, aún así, es insuficiente para erradicar el Aa del área subgingival. La terapia antibiótica sistémica si parece permitir la eliminación del Aa de las bolsas y del tejido gingival. Parece ser que la combinación de amoxicilina y metronidazol es el tratamiento más eficaz frente a esta bacteria, como se ha visto en estudios en pacientes con periodontitis juvenil localizada, periodontitis del adulto y periodontitis refractaria (61). Se ha evaluado la eficacia de la aplicación de antibióticos por vía tópica como las tetraciclinas o el metronidazol, pero no se han conseguido buenos resultados $(62,63)$. Se sugiere que el tratamiento más eficaz es el raspado y alisado radicular y posteriormente la realización de cirugía resectiva, junto con la administración de antibióticos por vía sistémica. 
La respuesta de $P g$ al tratamiento periodontal es parecida a la del Aa. Realizando raspado y alisado radicular se pueden disminuir temporalmente los niveles, pero no se consigue una erradicación definitiva, ya que esta bacteria suele localizarse en áreas de difícil acceso como las furcas o la base de las bolsas periodontales. En este caso la cirugía resectiva también es más predecible que la cirugía de acceso $(64,65,66)$. $\mathrm{El}$ tratamiento antimicrobiano sistémico es eficaz si se combina con raspado y alisado radicular y cirugía resectiva, por lo que éste es el tratamiento de elección para eliminar $\mathrm{Pg}$ del área subgingival.

En algunos casos, al cabo de seis meses del tratamiento periodontal, reaparecen los patógenos en la cavidad oral. Von Troil-Lindén realizó un estudio al respecto (67) en el que a los seis meses de realizar tratamiento periodontal, Aa reaparecía en un 33\% de los pacientes y $\mathrm{Pg}$ en un $43 \%$. Esto indica que es importante confeccionar un programa de mantenimiento adecuado para cada paciente, con el fin de evitar la recidiva de la enfermedad.

\section{ABSTRACT}

Among the bacteria related to periodontal disease, there are two species clearly associated to this disease: Actinobacillus actinomycetemcomitans and Porphyromonas gingivalis. This paper presents a review of the literature regarding this two periodontal pathogens, and showing their origin, prevalence, distribution, transmission and response to periodontal treatment.

\section{KEY WORDS}

Actinobacillus actinomycetemcomitans, Porphyromonas gingivalis, periodontal pathogen, periodontal destruction.

\section{CONCLUSIONES}

Los resultados de los trabajos revisados en este artículo sugieren, que existe una gran asociación entre $A a$ y $P g$ y la destrucción periodontal. No obstante, aún queda mucha investigación por delante para poder entender en profundidad estos microorganismos, su epidemiología y sus mecanismos patogénicos, aspectos que son importantes para establecer un diagnósti- co precoz y escoger la terapéutica adecuada. También son necesarias futuras investigaciones para establecer protocolos que permitan erradicar o disminuir los patógenos periodontales sin alterar la flora microbiana del huésped. Para ello habría que profundizar en campos como el tratamiento antibiótico, para determinar el tipo de fármaco y la dosis más eficaces, así como en la vía de administración y en la duración del tratamiento.

\section{BIBLIOGRAFÍA}

1. Brock TD, Madigan MT, Martinko JM, Parker J. Biology of microorganisms $7^{\text {th }}$ ed. Englewood Cliffs, New Jersey: Prentice-Hall International, Inc.; 1994, p.332-339, 397398, 406-409, 418-426, 438-444, 509-517.

2. Haffajee $A D$ Socransky SS. Microbial etiological agents of destructive periodontal diseases. Periodontol 2000 1994: 5: 78-111.

3. Slots J, Ting M. Actinobacillus actinomycetemcomitans and Porphyromonas gingivalis in human periodontal disease: ocurrence and treatment. Periodontol 2000 1999: 20: 82-121.

4. Bogert M, Bertold P, Brightman V, Craig R, DiRienzo J, Lai C-H, Lally E, Oler J, Ram C,Shenker B, Slots J, Taichman N, Tisot R. Longitudinal study of LJP families - two year surveillance. J Dent Res Annual meeting of AADR, 1989.

5. Ali RW, Velcescu C, Jivanescu MC, Lofthus B, Skaug N. Prevalence of 6 putative periodontal pathogens in subgingival plaque samples of Romanian adult periodontitis patients. J Clin Periodontol 1996: 23: 133-139.

6. Kamma JJ, Nakou M, Manti FA. Predominant microflora of severe, moderate and minimal periodontal lesions in young adults with rapidly progressive periodontitis. J Periodont Res 1995: 30: 66-72.

7. Socransky SS, Haffajee AD, Smith C, Dibart S. Relation of counts of microbial species to clinical status at the sampled site. J Clin Periodontol 1991: 18: 766-775.

8. Wolff LF, Aeppli DM, Pihlstrom B, Anderson L, Stoltenberg J, Osborn J, Hardie N, Shelburne C, Fischer G. Natural distribution of 5 bacteria associated with periodontal disease. J Clin Periodontol 1993: 20: 699706 .

9. Chen C, Slots J. Microbiological tests for Actinibacillus actinomycetemcomitans and Porphyromonas gingivalis. Periodontol 2000 1999: 20: 53-64. 
10. Ting M, Slots J. Microbiological diagnostics in periodontics. Compendium Contin Educ Dent 1997: 18: 861878. Translated into german' and published in Ästhet Zahnmed 1998: 3: 146-156.

11. Slots J. Selective medium for isolation of Actinobacillus actinomycetemcomitans. J Clin Microbiol 1982: 15: 606609.

12. Slots, J. Actinobacillus actinomycetemcomitans and Porphyromonas gingivalis in periodontal disease: introduction. Periodontol 2000 1999: 20: 7-13.

13. Van Winkelhoff AJ, Rams TE, Slots J. Sistemic antibiotic therapy in periodontics.Periodontol 2000 1996: 10: 45-78.

14. Genco RJ, Zambon JJ, Christersson LA. Use and interpretation of microbiological assays in periodontal diseases. Oral Microbiol Immunol 1986: 1: 73-79.

15. Genco RJ, Zambon JJ, Christersson LA. The origin of periodontal infections. Adv Dent Res 1988: 2: 245-259.

16. Slots J. Microbiology in periodontics. Tandlaegebladet 1986: 90: 794-798.

17. Genco RJ. Highlights of the conference and perspectives for the future. J Periodontol Res 1987: 22: 164-171.

18. Taubman MA, Genco RJ, Hillman JD. The specific pathogen-free human: a new frontier in oral infectious disease research. Adv Dent Res 1989: 3: 58-68.

19. Slots J, Van Wnkelhoff AJ. Antimicrobial therapy in periodontics. J Calif Dent Assoc 1993: 21: 51-56.

20. Gmür R, Guggenheim B. Interdental supragingival plaque - a natural habitat of Actinobacillus actinomycetemcomitans, Bacteroides forsythus, Campylobacter restus and Prevotella nigrescens. J Dent Res 1994: 73: 14211428.

21. Moore WEC, Moore LVH. The bacteria of periodontal diseases. Periodontol 2000 1994: 5: 66-77.

22. Asikainen S, Chen C. Oral ecology and person-to-person transmission of Actinobacillus actinomycetemcomitans and Porphyromonas gingivalis. Periodontol 2000 1999: 20: 65-81.

23. Kilian M, Prachyabrued W, Theilade E. Haemophili in developing dental plaque. Scand J Dent Res 1976: 84: 16-19.

24. Slots J, Gibbons RJ. Attachment of Bacteroides melaninogenicus subsp. assaccharolyticus to oral surfaces and its possible role in colonization of the mouth and of periodontal pockets. Infect Immun 1978: 19: 254-264.
25. Clerehugh V, Seymour GJ, Bird PS, Cullinan M, Drucker DB, Worthington HV. The detection of Actinobacillus actinomycetemcomitans, Porphyromonas gingivalis and Prevotella intermedia using an ELISA in an adolescent population with early periodontitis. J Clin Periodontol 1997: 24: 57-64.

26. Dahlén G, Manji F, Baelum V, Fejerskov O. Black-pigmented Bacteroides species and Actinobacillus actinomycetmcomitans in subgingival plaque of adult Kenyans. J Clin Periodontol 1989: 16: 305-310.

27. Ellen RP, Song M, Buivids IA. Inhibition of Actinomyces viscosus-Porphyromonas gingivalis coadhesion by trypsin and other proteins. Oral Microbiol Immunol 1992: 7: 198-203.

28. Ellwood R, Worthington HV, Cullinan MP, Hamlet S, Clerehugh V, Davies R. Prevalence of suspected periodontal pathogens identified using ELISA in adolescents of differing ethnic origins. J Clin Periodontol 1997: 24: 141-145.

29. Lamont RJ, Hersey SG, Rosan B. Characterization of the adherence of Porphyromonas gingivalis to oral streptococci. Oral Microbiol Immunol 1992: 7: 193-197.

30. Preus HR, Anerud A, Boysen H, Dunford RG, Zambon JJ, Loe $\mathrm{H}$. The natural history of periodontal disease. The correlation of selected microbiological parameters with disease severity in Sri Lankan tea workers. J Clin Periodontol 1995: 22: 674-678.

31. Asikainen S, Alaluusua S, Saxén L. Recovery of Actinobacillus actinomycetencomitans from teeth tongue and saliva. J Periodontol 1991: 62: 203-206.

32. Slots J, Reynolds HS, Genco RJ. Actinobacillus actinomycetencomitans in human periodontal disease: a crosssectional microbiological investigation. Infect Immun 1980: 29: 1013-1020.

33. Van Winkelhoff AJ, van der Velden U, Winkel EG, de Graaff J. Black-pigmented Bacteroides and motile organisms on oral mucosal surfaces in individuals with and without periodontal breakdown. J Periodont Res 1986: 21: $434-439$.

34. Wolff LF, Liljemark WF, Bloomquist CG, Philstrom BL, Schaffer EM, Brandt CL. The distribution of Actinobacillus actinomycetemcomitans in human plaque. J Periodont Res 1985: 20: 237-250.

35. Slots J, Feik D, Rams TE. Actinobacillus actinomycetencomitans and Bacteroides intermedius in human periodontitis age relationship and mutual association. J Clin Periodontol 1990: 17: 659-662. 
36. Van Steenbergen TJ, Petit MD, Scholte LH, van der Velden U, de Graaff J. Transmission of Porphyromonas gingivalis between spouses. J Clin Periodontol 1993: 20: 340-345.

37. Von Troil-Lindén B, Torkko H, Alaluusua S, JousimiesSomer H, Asikainen S. Salivary levels of suspected periodontal pathogens in relation to periodontal status and treatment.J Dent Res 1995: 14: 1789-1795.

38. Timmerman M; van der Weijden G, Armand S, Abbas F, Winkel $E$, van Winkelhoff $A$, van der Velden U. Untreated periodontal disease in Indonesian adolescents. Clinical and microbiological baseline data. J Clin Periodontol1998: 25: 215-224.

39. Müller HP, Lange DE, Müller RF. Actinobacillus actinomycetencomitans recovery from extracrevicular locations of the mouth. Oral Microbiol Immunol 1993: 8: 344-348

40. Müller HP, Zoller L, Eger T, Hoffmann S, Lobinsky D. Natural distribution of oral Actinobacillus actinomycetencomitans in young men with minimal periodontal disease. J Periodont Res 1996: 31: 373-380.

41. Socransky SS, Haffajee AD, Ximenez-Fyvie LA, Feres M, Mager D. Ecological considerations in the treatment of Actinobacillus actinomycetencomitans periodontal infections. Peridontol 2000 1997: 20: 341-362.

42. Ashimoto A, Chen C, Bakker I, Slots J. Polymerase chain reaction detection of 8 putaive pathogens in subgingival plaque of gingivitis and advanced periodontitis lesions. Oral Microbiol Immunol 1996: 11: 266-273.

43. Beck JD, Koch GG, Zambon JJ, Genco RJ, Tudor G. Evaluation of oral bacteria as risk indicators for periodontitis in older adults. J Periodontol 1992: 63: 93-99.

44. Christersson LA, Fransson CL, Dunford RG, Zambon JJ. Subgingival distribution of periodontal pathogenic microorganisms in adult periodontitis. J Periodontol 1992: 63: 418-425.

45. Gmür R, Guggenheim B. Monoclonal antibodies for the detection of 'periodontopathic' bacteria. Arch Oral Biol 1990: 35: 145S-151S.

46. Gmür R, Strub JR, Guggenheim B. Prevalence of Bacteroides forsythus and Bacteroides gingivalis in subgingival plaque of prosthodontically terated patients on short recall. J Peridont Res 1989: 24: 113-120.

47. Savitt ED, Socransky SS. Distibution of certain subgingival microbial species in selected periodontal conditions. J Periodont Res 1984: 19: 11-123.
48. White D, Mairand D. Association of oral Bacteroides with gingivitis and adult periodontitis. J Periodont Res 1981: 16: $259-265$.

49. Wolff LF, Aeppli DM, Philstrom B, Anderson L, Stoltenberg J, Osborn J, Hardie N, Shelburne C, Fischer G. Natural distribution of 5 bacteria associated with periodontal disease. J Clin Periodontol 1993: 20: 699-706.

50. Ebersole JL, Cappelli D; Sandoval MN. Subgingival distribution of Actinobacillus actinomycetencomitans in periodontitis. J Clin Periodontol 1994: 21: 65-75.

51. Torkko H, Asikainen S. Recovery of periodontal pathogens depends on sample transport time. J Dent Res 1996: 75: 241 .

52. Rams T, Flynn J, Slots J. Subgingival microbial associations in severe human periodontitis. Clin Infect Dis 1997: 25: S224-S226.

53. Lamster I, Kaluszhbner-Shapira I, Herrera-Abreu M, Sinha R, Grbic J. Serum IgG antibody response to Actinobacillus actinomycetencomitans and Porphyromonas gingivalis: implications for periodontal diagnosis. J Clin Peridontol 1998: 25: 510-516.

54. Aluusua S, Asikainen S, Lai CH. Intrafamilial transmission of Actinobacillus actinomycetencomitans. J Periodontol 1991: 62: 207-210.

55. Aluusua S, Saarela M, Jousimies-Somer H, Asikainen S. Ribotyping shows intrafamilial similarity in Actinobacillus actinomycetencomitans isolates. Oral Microbiol Immunol 1993: 8: 225-229.

56. Asikainen S, Chen C, Slots J. Likelihood of transmitting Actinobacillus actinomycetencomitans and Porphyromonas gingivalis in families with periodontitis. Oral Microbiol Immunol 1996: 11: 387-394.

57. DiRienzo JM, Slots J, Sixou M, Sol MA, Harmon R, McKay TL, Specific genetic variants of Actinobacillus actinomycetencomitans correlated with disease and health in a regional population of families with localizaed juvenile periodontitis. Infect Immun 1994: 62: 3058-3065.

58. Petit MD, van Steenbergen TJ, Scholte LM, van der Velden U, de Graaff J. Epidemiology and transmision of Porphyromonas gingivalis and Actinobacillus actinomycetencomitans among children and their family members. A report of 4 surveys. J Clin Periodontol 1993: 20: 641-650.

59. Saarela MH, Dogan B, Aluusua S, Asikainene S. Persistence of oral colonization by the same Actinobacillus actinomycetencomitans strain(s). J Periodontol 1999: 70: 504-509. 
60. Slots J, Rosling BG. Suppression of the periodontopathic microflora in localized juvenile periodontitis by systemic tetracycline. J Clin Periodontol 1983: 101: 465-486.

61. Van Winkelhoff AJ, Tijhof CJ, de Graaff J. Microbiological and clinical results of metronidazole plus amoxicillin theraphy in Actinobacillus actinomycetencomitans-associated periodontitis. J Periodontol 1992: 63: 52-57.

62. Goodson JM, Tanner A, McArdle S, Dix K, Watanabe SM. Multicenter evaluation of tetracycline fiber therapy III. Microbiological response. J Periodont Res 1991: 26: 440-451.

63. Hitzig C, Fosse T, Charbit Y, Bitton C, Hannoun L. Effects of combinated topical metronidazole and mechanical treatment on the subgingival flora in deep periodontal pockets in cuspids and bicuspids. J Periodontol 1997: 68: 613-617.
64. Mombelli A, Nyman S, Brägger U, Wennströnm J, Lang NP. Clinical and microbiological changes associated with an altered subgingival environment induced by periodontal pocket reduction.J Clin Periodontol 1995: 22: $780-787$.

65. Rosenberg ES, Torosian JP, Hammond BF, Cutler SA. Routine anaerobic bacterial culture and systemic antibiotic usage in the treatment of adult periodontitis: a 6year longitudinal study. Int J Periodontics Restorative Dent 1993: 13: 213-243.

66. Tuan M-C, Nowzari H, Slots J. Clinical and microbiological study of apically positioned flaps with and without osseus surgery. J Periodontol 1999: 70: 347.

67. Von Troil-Lindén B, Saarela M, Mattö J, Alaluusua S, Jousimies-Somer H, Asikainen S. Source of suspected periodontal pathogens re-emerging after periodontal treatment. J Clin Periodontol 1996: 23: 601-607. 
